\title{
An Infrequent Extraintestinal Manifestation of Ulcerative Colitis: Pulmonary Necrobiotic Nodules
}

\author{
Adam S. Myer ${ }^{1}$, Kushang Shah ${ }^{2}$, Kinner M. Patel ${ }^{3}$ \\ 1. Internal Medicine, Stony Brook University, State University of New York, Stony Brook, USA 2. Internal Medicine, \\ Stony Brook Medicine University Hospital, Stony Brook, USA 3. Pulmonary and Critical Care Medicine, Stony Brook \\ University Hospital, Stony Brook, USA
}

Corresponding author: Adam S. Myer, adam.myer@stonybrookmedicine.edu

\begin{abstract}
Pulmonary necrobiotic nodules are a rare extraintestinal manifestation (EIM) of inflammatory bowel disease (IBD), which are often overlooked when diagnosing cavitary pulmonary nodules. We present this case to highlight the importance of a thorough differential diagnosis, which includes EIMs of ulcerative colitis (UC), in this case as necrobiotic nodules. Herein, we present a 25 -year-old male patient with a history of poorly controlled UC who presented with fevers, left-sided abdominal pain, and bloody diarrhea. Imaging revealed cavitary pulmonary nodules without an infectious or malignant etiology. Lung biopsy and pathology confirmed a diagnosis consistent with necrobiotic nodules.
\end{abstract}

Categories: Internal Medicine, Gastroenterology, Pulmonology

Keywords: ulcerative colitis, inflammatory bowel disease, pulmonary nodules

\section{Introduction}

The two major forms of inflammatory bowel disease (IBD) are ulcerative colitis (UC) and Crohn's disease (CD). UC is a condition that affects the rectum and colon, characterized by inflammation of the mucosal layer in a continuous fashion [1]. CD can involve any portion of the GI tract, most commonly the ileocecal region, often with normal bowel in between diseased segments. Inflammation can be transmural, resulting in fistula formation.

Extraintestinal manifestations (EIMs) of IBD are seen in 25\%-40\% of IBD patients [2]. The most common organ systems affected are musculoskeletal, dermatologic, ocular, and hepatobiliary [3]. Pulmonary involvement is rare, and the true prevalence is unknown [4]. Pulmonary manifestations include a wide variety of patterns, such as upper airway (glottis and subglottic edema), large airway (bronchiectasis), small airway (bronchiolitis obliterans syndrome), pulmonary vasculature and thromboembolic disease, and lung parenchymal disease [5]. One pattern of lung parenchymal disease associated with IBD is necrobiotic

Received 07/14/2020 Review began $07 / 31 / 2020$ Review ended 08/11/2020 Published 08/16/2020

() Copyright 2020 Myer et al. This is an open access article distributed under the terms of the Creative Commons Attribution License CC-BY 4.0., which permits unrestricted use, distribution, and reproduction in any medium, provided the original author and source are credited. nodules, which requires a thorough workup because of its resemblance to other autoimmune and infectious etiologies.

\section{Case Presentation}

A 25-year-old male patient with a history of UC presented with fevers, chest soreness, left-sided abdominal pain associated with urgency, and 15-20 episodes per day of bloody diarrhea. CT of the chest, abdomen, and pelvis did not show any evidence of colitis but did reveal multiple necrotic, cavitary pulmonary nodules (Figure 1), and the patient was started on IV vancomycin, cefepime, and Flagyl. The patient was diagnosed with UC two years prior and had been on mesalamine along with steroid suppositories, which he was noncompliant with. The patient denied any history of drug abuse, cardiovascular disease, or dental procedures done in the recent past. Social history was significant for former eight pack-year history of cigarettes smoking and vaping, which he stopped one month prior to presentation. 


\section{Cureus}

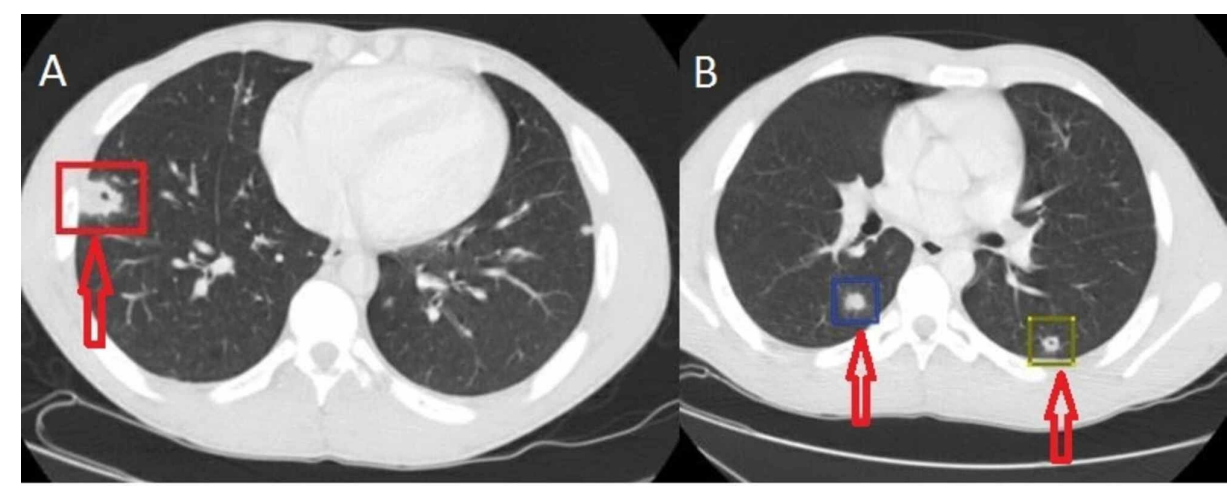

FIGURE 1: CT of the chest with IV contrast showing several scattered lung nodules with evidence of necrosis and cavitation. The largest nodule ( $A$, red square) is seen in the right lower lobe and does demonstrate necrosis and cavitation, measuring $2 \mathrm{~cm}$. Also seen are several other smaller lung nodules ( $B$, blue and yellow squares).

On admission, the patient was normotensive, afebrile, and saturating $>96 \%$ on room air. On physical exam, there were coarse breath sounds, tenderness to palpation in bilateral lower quadrants of the abdomen with no rebound tenderness, no cardiac murmurs, Janeway lesions, Osler nodes, or Roth spots. Laboratory markers showed normal chemistry and a hemoglobin of $8.7 \mathrm{~g} / \mathrm{dL}$ with a mean corpuscular volume (MCV) of 72.9 , platelet count of $352 \mathrm{~K} / \mu \mathrm{L}$, white blood cell (WBC) $11.35 \mathrm{~K} / \mu \mathrm{L}$, erythrocyte sedimentation rate (ESR) of $64 \mathrm{~mm} / \mathrm{hr}$, C-reactive protein (CRP) of $3.5 \mathrm{mg} / \mathrm{dL}$, and fecal calprotectin $580 \mu \mathrm{g} / \mathrm{g}$. Infectious etiologies were negative for HIV, hepatitis B, histoplasmosis, coccidioidomycosis, blastomycosis, Coxiella burnetii, Brucella, Clostridium difficile, Aspergillus, and Lyme disease. Acid-fast bacillus (AFB) culture from the sputum revealed no AFB, and stool culture showed no ova and parasite growth.

A transthoracic echocardiogram (TTE) was performed, which showed a possible tissue density on the pulmonic valve. Subsequently, a cardiac MRI (Figure 2) was performed to further characterize the pulmonic valve and did not show any vegetations. Blood cultures showed no growth of organisms. Autoimmune workup for granulomatosis with polyangiitis, microscopic polyangiitis, and rheumatoid arthritis was negative along with negative anti-citrullinated protein antibody (anti-CCP) and rheumatoid factor (RF). Antinuclear antibody (ANA) positivity titer was 1:80, which was deemed to be insignificant in our patient. Antibiotics were discontinued, as the concern for infection remained low. 


\section{Cureus}

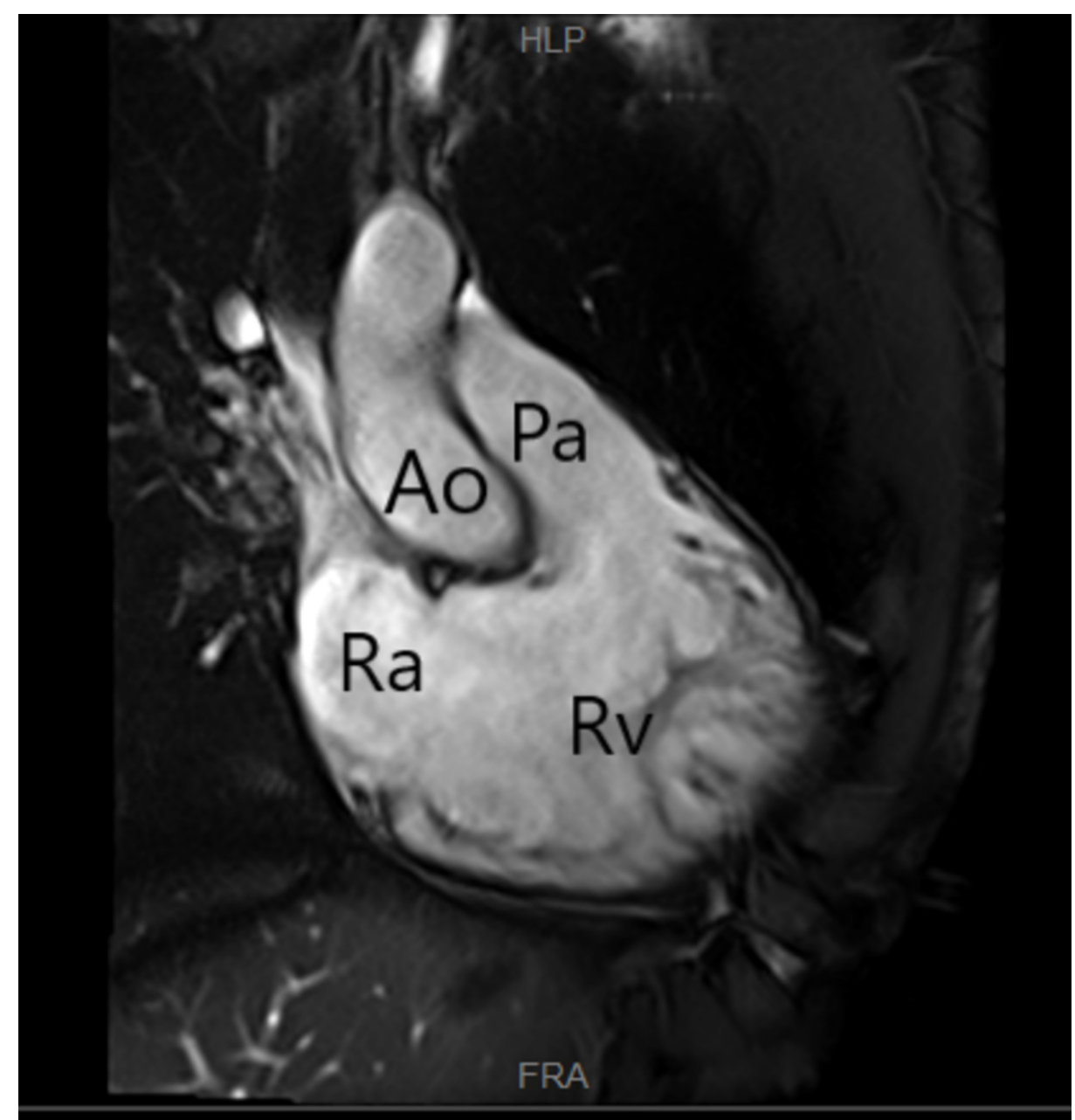

FIGURE 2: Cardiac MRI white blood imaging, coronal view showing right atrium (Ra), right ventricle (Rv), pulmonary artery $(\mathrm{Pa})$, and aorta (Ao). No evidence of intracavitary thrombus or valvular vegetation.

CT-guided lung biopsy was done, which revealed fibrosis with mixed inflammatory infiltrate containing macrophages, lymphocytes, scatter neutrophils, and plasma cells (Figure 3). Mesalamine was stopped as the patient noticed return of abdominal pain with it. Furthermore, case studies have shown pulmonary infiltrates with related 5-aminosalicylic acid derivatives [6]. The patient was treated with $40 \mathrm{mg}$ oral prednisone once a day that was tapered by $10 \mathrm{mg}$ once a week and transitioned to adalimumab. Symptoms gradually improved with therapy.

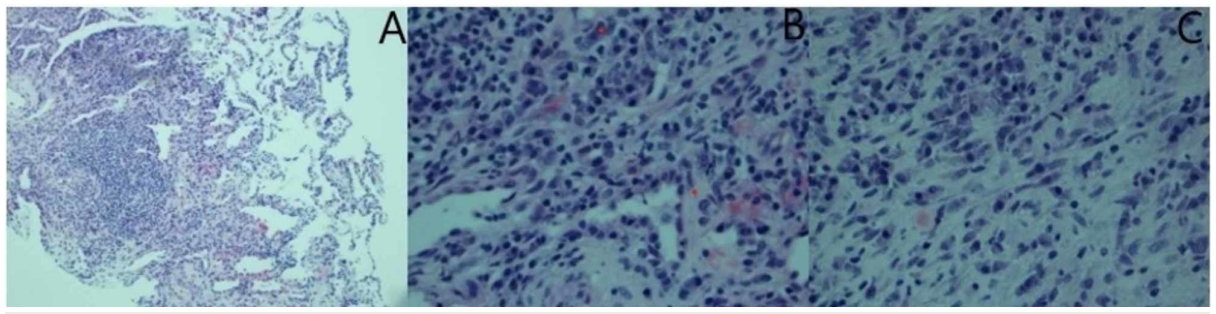

FIGURE 3: Lung tissue from CT-guided lung biopsy demonstrating fibrosis with mixed inflammatory inflitrate, consisting of macrophages, lymphocytes, scattered neutrophils, and plasma cells. Image A (not zoomed) and images B and C (magnified). 
Our case describes a rare case of necrobiotic pulmonary nodules in a patient with poorly controlled UC. Necrobiotic nodules are sterile nodules composed of inflammatory cells with necrosis [7]. On imaging, these nodules can appear round and well defined, exhibiting cavitation [5]. The pathophysiology of pulmonary EIM in IBD patients is not entirely known; however, both the respiratory and intestinal mucosa are derived from the primitive foregut. Both epithelia contain submucosal lymphoid tissue, and exposure to antigens via inhalation or ingestion can trigger inflammation. Another hypothesis suggests that lung involvement in IBD can be due to systemic inflammatory mediators released from inflamed bowel mucosa [8].

The appearance of these necrobiotic nodules can mimic other cavitating diseases, making them a diagnostic challenge. Before making this diagnosis, a pertinent, yet thorough differential (Table 1) that includes infectious, autoimmune, vasculitis and malignant etiologies must be excluded. On initial presentation, there was concern for an infectious process causing septic emboli to the lungs. However, the blood cultures revealed no growth and low infectious markers, and unrevealing cardiac MRI ruled out the possibility of an infectious etiology. Necrobiotic nodules may also be seen in rheumatoid arthritis and granulomatosis with polyangiitis; however, RF, anti-CCP, and antineutrophil cytoplasmic antibody (ANCA) were negative [4]. Lung biopsy was then done, which revealed histologic confirmation of necrobiotic nodules.

\author{
Differential Specific Diagnosis \\ Autoimmune Rheumatoid arthritis, pyoderma gangrenosum, sarcoidosis, inflammatory bowel disease \\ Vasculitis Granulomatosis with polyangiitis, Behcet's disease \\ Malignancy Primary lung (squamous cell carcinoma, adenocarcinoma), lymphoma, metastatic disease \\ Septic emboli, lung abscess (Streptococcus, Staphylococcus, Klebsiella), Mycobacterium tuberculosis, \\ Infectious Mycobacterium avium complex, aspergillosis, histoplasmosis, blastomycosis,coccidioidomycosis, \\ paracoccidioidomycosis \\ Cystic Langerhans' cell histiocytosis, lymphangioleiomyomatosis, idiopathic pulmonary fibrosis
}

TABLE 1: Differential diagnosis of cavitating lung nodules.

In review of the literature, there are several case reports of pulmonary necrobiotic nodules in both UC and $\mathrm{CD}$ patients; however, the absolute incidence is unknown. Per our review, there are more documented cases of necrobiotic nodules in CD compared to UC. There are two reported cases of pulmonary necrobiotic nodules in UC: a 70-year-old male with UC, who presented with hemoptysis and found to have cavitary pulmonary nodules; biopsy confirmed necrobiotic nodules. The patient was treated with a two-week course of $60 \mathrm{mg}$ prednisone daily and subsequent imaging showed resolution [9]. The second case is of a 17-yearold male diagnosed with UC and primary sclerosing xholangitis (PSC) two years prior in remission, who presented with cough and chest pain. Imaging showed pulmonary nodules, which were biopsied and consistent with necrobiotic nodules. The patient was treated with prednisone and follow-up CT showed greater than $75 \%$ reduction of nodule size [10]. There was also a case of a 22 -year-old female with UC who had cavitary pulmonary nodules on imaging; however, they were not biopsied [11]. Although the majority of case reports show improvement of pulmonary EIM with steroid therapy, there are no guidelines on duration, dosage, and follow-up imaging [10].

\title{
Conclusions
}

In IBD patients presenting with pulmonary nodules, it is important to keep necrobiotic nodules as a differential. Etiologies such as autoimmune, infectious, and malignancy should be excluded before coming to this diagnosis. The necrobiotic nodules present in IBD seem to respond to steroid therapy; however, further studies are indicated to determine the duration, dosage, and follow-up testing needed.

\section{Additional Information \\ Disclosures}

Human subjects: Consent was obtained by all participants in this study. Conflicts of interest: In compliance with the ICMJE uniform disclosure form, all authors declare the following: Payment/services info: All authors have declared that no financial support was received from any organization for the submitted work. Financial relationships: All authors have declared that they have no financial relationships at present or within the previous three years with any organizations that might have an interest in the submitted work. Other relationships: All authors have declared that there are no other relationships or activities that could appear to have influenced the submitted work. 


\section{Cureus}

\section{References}

1. Hendrickson BA, Gokhale R, Cho JH: Clinical aspects and pathophysiology of inflammatory bowel disease . Clin Microbiol Rev. 2002, 15:79-94. 10.1128/cmr.15.1.79-94.2002

2. Bernstein CN, Blanchard JF, Rawsthorne P, Yu N: The prevalence of extraintestinal diseases in inflammatory bowel disease: a population-based study. Am J Gastroenterol. 2001, 96:1116-1122. 10.1111/j.1572-0241.2001.03756.x

3. Levine JS, Burakoff R: Extraintestinal manifestations of inflammatory bowel disease . Gastroenterol Hepatol (N Y). 2011, 7:235-241.

4. Ji XQ, Wang LX, Lu DG: Pulmonary manifestations of inflammatory bowel disease . World J Gastroenterol. 2014, 20:13501-13511. 10.3748/wjg.v20.i37.13501

5. Cozzi D, Moroni C, Addeo G, et al.: Radiological patterns of lung involvement in inflammatory bowel disease. Gastroenterol Res Pract. 2018, 2018:5697846. 10.1155/2018/5697846

6. Huang PH, Kuo CJ, Lin CW, et al.: Mesalazine-related lung disease in a patient with ulcerative colitis: a case report. Medicine. 2018, 97:e13242. 10.1097/MD.0000000000013242

7. El-Kersh K, Fraig M, Cavallazzi R, Saad M, Perez RL: Pulmonary necrobiotic nodules in Crohn's disease: a rare extra-intestinal manifestation. Respir Care. 2014, 59:e190-e192. 10.4187/respcare.03176

8. Spira A, Grossman R, Balter M: Large airway disease associated with inflammatory bowel disease . Chest. 1998, 113:1723-1726. 10.1378/chest.113.6.1723

9. Barril S, Rodrigo-Troyano A, Gimenez A, Sibila O: Migratory pulmonary nodules in a patient with ulcerative colitis. (Article in En, Spanish). Arch Bronconeumol. 2015, 51:303-304. 10.1016/j.arbres.2014.07.006

10. Barbosa M, Gondim F, Vosqui F, et al.: P-15 pulmonary necrobiotic nodules in a patient with UC and primary sclerosing cholangitis: a rare extraintestinal manifestation of IBD: case report. Inflam Bowel Dis. 2012, 18:S19. 10.1097/00054725-201212001-00048

11. Kellish A, Soal V, Caskey E, Hassinger G, Terrigno N: Pulmonary necrobiotic nodules at time of diagnosis in a patient with ulcerative colitis. Cureus. 2020,12:e7474. 10.7759/cureus.7474 